\title{
The short term effect of cyclic passive stretching on plantarflexor resistive torque after acquired brain injury
}

\author{
BJ Singer ${ }^{1}$ JW Dunne ${ }^{2}$ KP Singer ${ }^{1}$
}

${ }^{1}$ Centre for Musculoskeletal Studies, The University of Western Australia, Perth.

${ }^{2}$ Department of Neurology, Royal Perth Hospital, Perth, Western Australia.

Address for corresponding author:

Dr BJ Singer, PT, $\mathrm{PhD}$

Centre for Musculoskeletal Studies,

Level 2, Medical Research Foundation building,

Royal Perth Hospital

Rear 50 Murray Street WA 6000.

Australia

Email: bjs@cms.uwa.edu.au

Phone: +61 892240229

Fax: +61 892240204 


\section{Abstract}

Background: Increased calf muscle stiffness is a common impairment following acquired brain injury. This study examined the immediate effects of cyclic ankle stretching at two stretch velocities on calf stiffness in individuals with hemiparesis $(n=17)$ and control subjects $(n=10)$. Methods: Cyclic ankle stretching was applied for three minutes at velocities of $5^{\circ} \mathrm{sec}^{-1}$ and $25^{\circ}$ $\sec ^{-1}$ using a purpose-built dynamometer. Surface electromyography was employed to ensure stretches were passive. Peak plantarflexor resistive torque was derived from torque-angle curves. Comparisons were made between groups, velocities and between limbs for hemiparetic subjects. Findings: At baseline, mean peak plantarflexor resistive torque was greater in the affected limbs of hemiparetic subjects than their contralateral limbs ( $\mathrm{p}<0.001$ ); however there was no significant difference between groups. Plantarflexor resistive torque was reduced in all limbs following cyclic stretching regardless of stretch velocity $(p<0.005)$. Two distinct patterns of response were observed in hemiparetic subjects. In nine cases the affected limb responses did not differ from the contralateral limb or control data. In the remaining eight cases mean peak plantarflexor resistive torque in the affected limb was greater than the contralateral limb and control values. In this subgroup, peak plantarflexor resistive torque was significantly affected by stretch velocity and showed the greatest reduction following cyclic stretching.

Interpretation: Cyclic stretching has been shown to produce a short term reduction in calf stiffness in a subgroup of individuals with hemiplegia. Further investigation is required to elaborate the characteristics of those most likely to respond optimally to this intervention.

Keywords: skeletal muscle; spasticity; hypertonia; passive resistive torque; ankle; acquired brain injury

Word count $=246$ words 


\section{Introduction}

Hypertonia, or increased resistance to lengthening (Stolov 1966), in the calf muscles is a common impairment after acquired brain injury (ABI) which can have significant consequences for functional mobility. Muscles that are excessively resistant to passive lengthening, or that lengthen more slowly than usual due to abnormal stiffness, may prevent achievement of adequate range of dorsiflexion motion. Increased calf stiffness is likely to impact particularly on tasks that require momentum, such as rising from a chair or ankle dorsiflexion during the swing phase of gait. Following acquired central nervous system injury, resistance that increases with the velocity of stretch has traditionally been considered to be due to stretch induced contraction associated with hyper-active tonic stretch reflexes (spasticity) (Lance 1980). However there are a number of other factors that can contribute to increased resistance to passive lengthening of the calf muscles (Gajdosik 2001, Singer et al 2001). For instance, in a classic study of patients with ABI conducted by Dietz and colleagues (1981), failure to adequately lengthen the plantarflexors during the swing phase of gait was found to be due to increased stiffness in these muscles rather than stretch reflex induced contraction or failure of the dorsiflexors to generate sufficient torque. Impaired visco-elastic behavior has also been demonstrated in the calf muscles of chronic stroke patients (Lamontagne et al 2000) and older women (Gajdosik et al 2005). Abnormalities in actinmyosin crosslinks, either due to a greater than normal number of actin-myosin crosslinks, or a failure of these weakly bound links to disengage with passive stretch, have also been postulated to contribute to hypertonia (Hufschmidt \& Mauritz 1985, Carey \& Burghart 1993). Singer et al (2003) provided evidence for increased resistance to passive stretch in hemiparetic subjects that was greater at faster rates of stretch but was not related to stretch reflex induced contraction.

In clinical practice, both sustained and cyclic stretching paradigms have been used to reduce calf muscle stiffness. Repetitive motion (jogging) has been shown to be more effective than static holds in reducing stiffness at the ankle joint in healthy subjects (McNair \& Stanley, 1996); however relaxation of peak tension was optimised by short duration sustained stretches (McNair et al., 2000).

In the management of orthopedic surgical interventions, continuous passive motion (CPM) has been utilized for many years to maintain the health of articular cartilage, prevent the development of joint stiffness and reduce limb edema post surgery (O'Driscoll \& Giori 2000). The potential for CPM to reduce soft tissue stiffness in individuals with hypertonia following ABI has only been 
explored fairly recently. A few studies have examined the utility of cyclic stretching to produce short term improvement in ankle dorsiflexion range of motion as well as calf muscle stiffness in subjects with hypertonia following ABI (Bressel and McNair, 2002; Zhang et al., 2002; Yeh et al., 2007). In addition to achieving these aims, CPM was found to better tolerated than static stretching in this patient group (Yeh et al., 2007). Previous studies have utilised periods of between 30 and 45 minutes of cyclic stretching (Bressel and McNair, 2002; Zhang et al., 2002; Yeh et al., 2007), despite the fact that almost 50\% of the overall reduction in passive resistance has been shown to occur within the first five minutes of cyclic stretching (Yeh et al 2007). In clinical settings, therapists, trainers, or even patients themselves frequently apply brief passive stretches to 'loosen up’ muscles prior to commencing activity. The aim of this study was to compare the immediate effect of three minutes of cyclic ankle stretching on peak plantarflexor resistive torque (PPRT) in individuals with adult onset hemiparesis and control subjects. A secondary aim was to compare the effect of stretch velocity on PPRT.

\section{Methods}

\subsection{Subjects}

A sample of convenience of 17 individuals with hemiparesis following stroke or traumatic brain injury comprising nine females and eight males, with a mean age of 45 (range 22-76) and 10 normal controls with a similar age range (mean age 37, range 20-56, seven females and three males) were studied. The mean time since onset for subjects with hemiparesis was 11.4 months (range 6 - 36 months). Hemiparetic subjects were included if: they had clinical evidence of hypertonia of the affected calf muscle; at least $5^{\circ}$ of ankle dorsiflexion was able to be achieved by passive stretching with the knee flexed, and they were able to consent to and co-operate with the test procedure. All subjects signed written consent prior to testing. The investigation received institutional ethics approval.

\section{$2.2 \quad$ Procedures}

Subjects were positioned in supine lying with both legs supported in flexion (Figure 1). The test limb was firmly secured in the device and the centre of rotation of the footplate was aligned as closely as possible with the axis of rotation of the ankle joint (Inman 1976). Cyclic stretches were applied for three minutes at two separate stretch velocities $\left(5^{\circ} \mathrm{sec}^{-1}\right.$ and $\left.25^{\circ} \mathrm{sec}^{-1}\right)$ using a computer controlled dynamometer. The stretch excursion was from $20^{\circ}$ plantarflexion to $80 \%$ of each subject's maximal dorsiflexion range. The right leg was tested for all control subjects. In subjects with hemiparesis, the less affected limb was tested first to allow for familiarization with 
the test procedure. Surface electromyography from the medial soleus and tibialis anterior muscles was sampled in all subjects to ensure stretches were passive. The skin underlying the electrodes was cleaned using 70\% ethanol and an abrasive paste to reduce impedance. The area was shaved if required. Pairs of $10 \mathrm{~mm}$ disposable $\mathrm{Ag} / \mathrm{AgCl}$ electrodes were placed on the medial soleus and tibialis anterior muscle bellies with an inter-electrode distance of $25 \mathrm{~mm}$. The ground electrode was placed on the lateral malleolus. The gain was set to detect any volitional or stretch induced contraction. Data were displayed during collection and any test cycle demonstrating muscle activity was discarded. Cyclic stretches at $5^{\circ} \sec ^{-1}$ were applied for three minutes followed by three minutes of stretches at $25^{\circ} \mathrm{sec}^{-1}$. A rest period of at least 60 seconds was employed between test velocities; however the subject remained secured in the device until the procedure was completed.

\subsection{Data reduction and analysis}

Raw torque, angle (position) and EMG data were sampled at $1000 \mathrm{~Hz}$ and stored to disc. Torque and angle data were calibrated and filtered (fourth order Butterworth low pass 6Hz) with zero offsets removed to create torque-angle curves. Peak plantarflexor resistive torque was represented by the torque (N.m) at $80 \%$ of maximal passive range of ankle dorsiflexion. Peak plantarflexor resistive torque at baseline was compared with data following three minutes of cyclic stretching for hemiplegic and control subjects and between limbs in the former group using paired and unpaired 't'-tests, and also between stretch velocities for each group. A probability of $\mathrm{p}<0.05$ was used as the criterion for determining meaningful differences between limbs and groups.

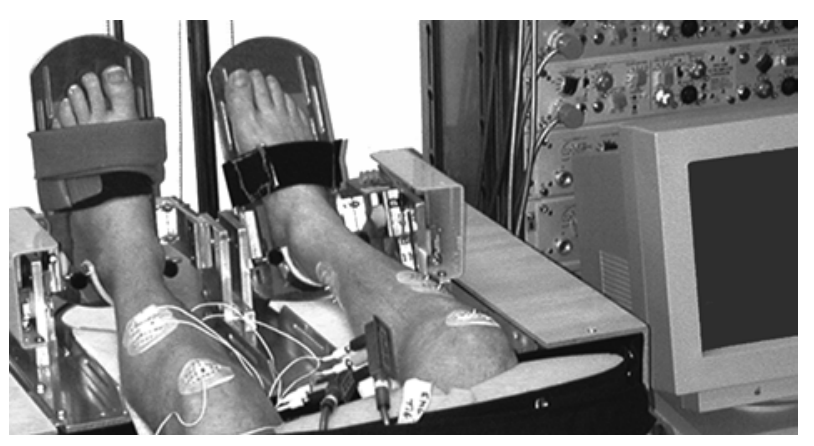

Figure 1 - A customized dynamometer was used to evaluate resistance to passive movement of the ankle from $10^{\circ} \mathrm{PF}$ to $80 \%$ of each subject's maximal DF range. Surface EMG from the medial soleus and tibialis anterior muscles was sampled to ensure stretches were passive. 


\section{Results}

Data are depicted graphically in figures 2\&3. Mean baseline PPRT in the affected limbs of hemiparetic subjects was greater than their contralateral limbs $(\mathrm{p}<0.001)$ but was not significantly different from normal subjects (Figure 2). There was considerable within group variation (Figures 2\&3). The affected limb of hemiparetic subjects showed significantly greater resistance to fast compared with slow stretches $(\mathrm{p}<0.004)$. A significant reduction in PPRT was observed in all limbs following cyclic stretching with no difference between stretch velocities (control limbs and affected limb of hemiplegic subjects $\mathrm{p}<0.005$; less affected leg $\mathrm{p}<0.02$ )(Figure 2). Mean PPRT for the less affected limb in the group with hemiparesis was lower than values in normal subjects at baseline and showed minimal reduction in PPRT over repeated cyclic stretches (Figure 2). Planned comparison of responder types revealed two distinct trends in the affected limbs of hemiplegic subjects (Figure 3). In Group A (n=9) responses were not significantly different between limbs nor from normal subject data. In Group B $(n=8)$ the affected limb was significantly stiffer than all other groups (contralateral $\operatorname{limb} \mathrm{p}=0.01$, controls $\mathrm{p}=0.014$ ), a marked velocity dependent effect was evident and PPRT was significantly reduced by cyclic stretching $(\mathrm{p}=0.026$ at $5^{\circ} \sec ^{-1}, \mathrm{p}=0.005$ at $25^{\circ} \mathrm{sec}^{-1}$ )(Figure 3 ).

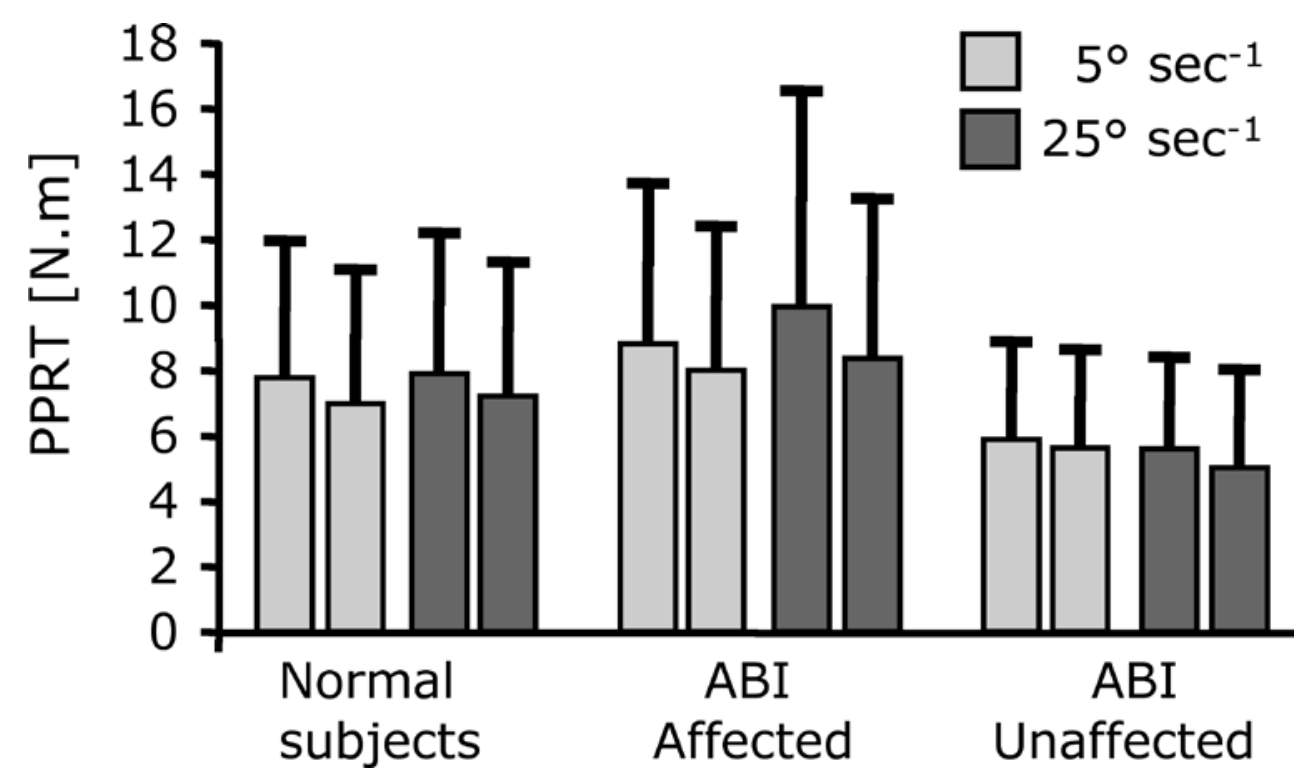

Figure 2 - Mean peak plantarflexor resistive torque $( \pm \mathrm{SD})$ for subjects with adult onset hemiparesis $(n=17)$ and age matched controls $(n=10)$ at baseline and following three minutes of cyclic stretching at $5 \% \mathrm{sec}^{-1}$ and $25 \% \mathrm{sec}^{-1}$ respectively. 


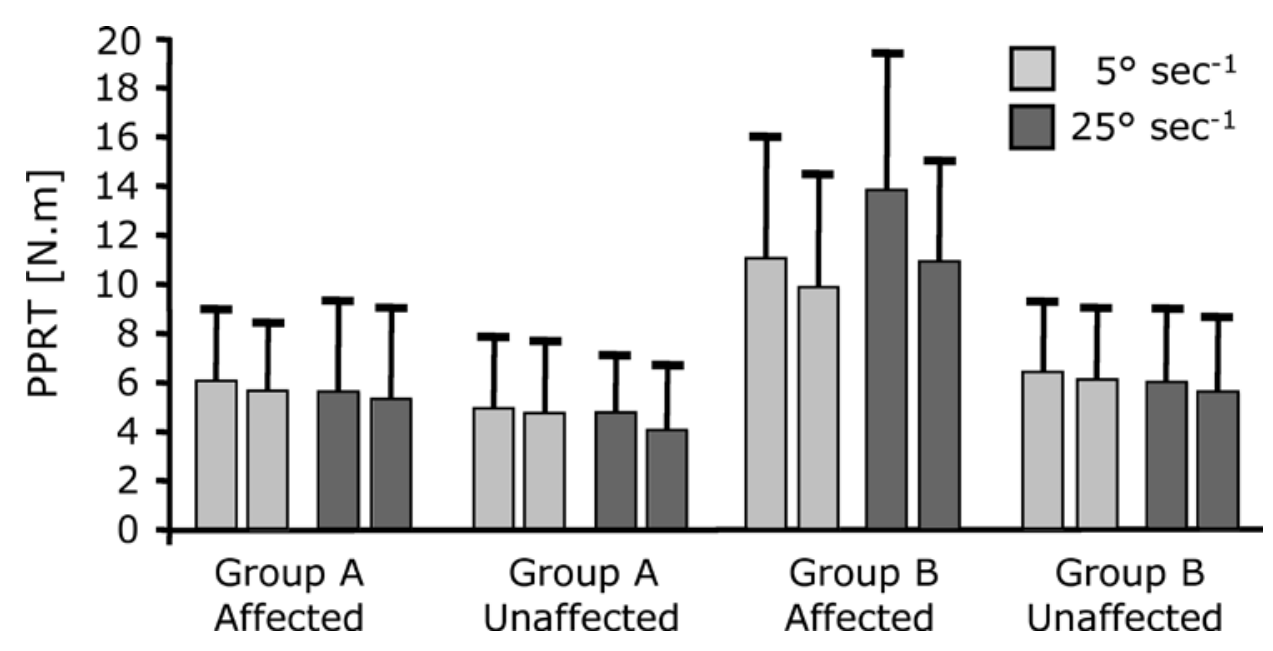

Figure 3 - Post hoc analysis identified two types of response in subjects with hemiparesis. Data are presented for mean peak plantarflexor resistive torque $( \pm S D)$ in the affected and unaffected limbs of Group A ( $n=9)$ and Group B ( $n=8)$ at baseline and following three minutes of cyclic stretching at $5 \% / \mathrm{sec}^{-1}$ and $25^{\circ} / \mathrm{sec}^{-1}$. Normal data are not depicted in this graph [refer to fig 2]. The affected limb of Group B subjects was significantly stiffer than all other limbs, demonstrated a marked effect of stretch velocity and significantly reduced PPRT after 3 minutes compared with all other limbs.

\section{Discussion}

The aim of this scoping investigation was to examine the potential for cyclic stretching of the ankle to produce a reduction in calf muscle stiffness in individuals with adult onset hemiparesis. The immediate effect of a brief period of cyclic ankle stretching on PPRT, as well as the effect of stretch velocity, were examined in a small group of subjects with hemiparesis secondary to ABI and neurologically normal individuals. The period of cyclic stretching was shorter than previous investigations (Bressel and McNair, 2002; Zhang et al., 2002; Yeh et al., 2007). In the study by Yeh et al (2007) 50\% of the overall reduction in passive resistance occurred within the first five minutes of cyclic stretching. Short periods of cyclic stretching, if shown to reduce resistance to passive stretch, could have considerable clinical utility.

The response to cyclic stretching and the influence of stretch velocity on the less affected leg of the whole cohort with ABI was not significantly different to controls. Surprisingly, mean PPRT for the less affected leg was lower than control values (Figure 2). This may be due to disuse atrophy as most of the hemiparetic subjects studied were relatively sedentary (Stein \& Wade 
2005) as well as a potential influence from age related sarcopenia (Narici \& Maganaris 2005) as the hemiparetic group were slightly older than the control subjects.

Two different types of response were demonstrated in the affected limb of the hemiparetic subjects. In some individuals (Figure 3 - Group A) PPRT was not significantly different to control subjects, there was minimal reduction in PPRT following cyclic stretching, and the result was not affected by stretch velocity. In these subjects, the clinically evident hypertonia may have been predominantly due to an increase in the proportion of collagen to muscle fibres known to be associated with inactivity (Williams \& Goldspink 1984). In animal models, immobilisation has been shown to result in relative dehydration of the connective tissue making up ligaments and joint capsule, and reduced lubrication between collagen fibres resulting in micro-adhesions (Trudel and Uhthoff, 2000); all of which could result in increased resistance to passive lengthening. Theoretically such changes in muscle and tendon stiffness could be improved via cyclic movement; however in the present study the duration of the stretching may have been insufficient to increase soft tissue lubrication, and the excursion of the stretch may not have effected sufficient collagen deformation to reduce resistance to passive movement.

In the 'responder' group of subjects with hemiparesis marked velocity dependent passive stiffness was demonstrated in the affected limb (Figure 3 - Group B). The greatest reduction in PPRT after 3 minutes cyclic stretching was demonstrated at a stretch velocity of $25^{\circ} \sec ^{-1}(\mathrm{p}<0.005)$. Improved soft tissue lubrication producing easier sliding of filaments may have contributed to the reduction in PPRT following cyclic stretching seen in all subjects; however this would not be expected to be influenced by stretch velocity. The precise mechanisms contributing to the reduction in PPRT following a period of cyclic stretching are unable to be elucidated from this investigation.

No formal neurological evaluation was undertaken, nor were demographic data collected which might have allowed the clinical features associated with the different types of response in hemiparetic subjects to be elaborated. Further investigation is required to examine the association between diagnosis (type, severity and location of brain injury), clinical presentation and response to cyclic stretching in order to identify the characteristics of those who might benefit from this intervention. Future studies are also needed to examine the effect of longer periods of cyclic stretching and to investigate the latency of any beneficial effect of cyclic stretching. 
There were a number of limitations on this pilot investigation, the most important being that only a small number of subjects were studied and they may not represent the larger population of survivors of ABI. Subjects were included in this investigation if they had hemiparesis due to adult onset ABI, clinically evident hypertonia and no major ankle contracture. Subjects with traumatic brain injury as well as stroke were included in the ABI cohort with an average age of 45 years. This is considerably younger than many cohorts with hemiparesis secondary to stroke, although several other studies looking at the effect of cyclic stretching have examined subjects with similar age range (Zhang et al., 2002; Yeh et al., 2007). Previous investigators have reported differences in passive viscoelastic properties in the calf muscles of older versus younger women (Gajdosik et al 2005). The applicability of these findings to the wider population with chronic hemiparesis, particularly older adults, is unknown.

The potential effect of co-morbidities on muscle stiffness was not investigated in the present study. In particular the presence of diabetes mellitus in the cohort studied was not documented, which may have confounded these data as an increase in plantarflexor muscle stiffness has been demonstrated in some individuals with diabetes mellitus (Trevino et al 2004, Rao et al 2006).

The fixed order of stretch velocity may also have influenced the data as previous studies have shown the potential for initial stretches to reduce the resistance to subsequent stretches (Bressel \& McNair 2002, Chung et al 2004, Yeh et al 2007). Stretches at a velocity too slow to induce reflex contraction were performed first, in part to minimize the risk of injury to the calf muscles. A short rest period was provided between the two series of stretches at different velocities. It would be expected that any thixotropic effects of the initial slow stretches would have reduced the resistance to subsequent stretches. However, in Group B the greatest resistance was evident in the affected leg at $25^{\circ} \mathrm{sec}^{-1}$ despite the prior three minutes of slow stretching (Figure 3).

Increased passive muscle stiffness has been reported previously in individuals with hypertonia (Dietz et al 1981, Lamontagne et al 2000, Singer et al 2003) and is postulated to be an adaptive mechanism to compensate for impaired distal control (Carey \& Burghart 1993, Dietz et al 1983). Passive calf muscle stiffness contributes towards the total plantarflexor moment and assists ankle push-off at the end of the stance phase (Lamontagne et al., 2000). Increased stiffness may confer additional stability during gait and in response to sudden external perturbations in individuals with hemiplegia (Dietz et al 1983). However this adaptation may have a negative impact on movements which require rapid passive lengthening of the plantarflexor muscles, such as rising 
from a seated position or dorsiflexing the foot rapidly during walking. Reducing velocity dependent stiffness immediately prior to functional activity may improve ease of mobility and has the potential to reduce the risk of falls due to tripping.

\section{Conclusions:}

Increased velocity dependent passive stiffness was demonstrated in the affected limb of a subset of individuals with chronic hemiparesis. The potential for cyclic stretching to have a short-term benefit for some individuals with increased calf muscle stiffness following acquired brain injury is supported by these pilot data. Further investigation is required to elaborate the characteristics of those most likely to respond optimally to this intervention.

Word count $=2521$ words, 3 figures . 


\section{References}

Bressel, E., McNair, P.J., 2002. The effect of prolonged static and cyclic stretching on ankle joint stiffness, torque relaxation, and gait in people with stroke. Phys. Ther. 82, 880-7.

Carey, J.R., Burghardt, T.P., 1993. Movement dysfunction following central nervous system lesions: a problem of neurologic or muscular impairment? Phys. Ther. 73, 538-47.

Chung, S.G., van Rey, E., Bai, Z., Roth, E.J., Zhang, L-Q., 2004. Biomechanic changes in passive properties of hemiplegic ankles with spastic hypertonia. Arch. Phys. Med. Rehabil. 85, 1638-46.

Dietz, V., Quintern, J., Berger, W., 1981. Electrophysiological studies of gait in spasticity and rigidity. Evidence that altered mechanical properties of muscle contribute to hypertonia. Brain. 104, 431-49.

Dietz, V., Quintern, J., Berger, W., 1983. Normal and impaired regulation of muscle stiffness in gait: a new hypothesis about muscle hypertonia. Exp. Neurol. 79, 680-7.

Gajdosik, R.L.. 2001. Passive extensibility of skeletal muscle: review of the literature with clinical implications. Clin Biomech.16, 87-101

Gajdosik, R.L., Vander Linden, D.W., McNair, P.J., Riggin, T.J., Albertson, J.S., Mattick, D.J., Wegley, J.C., 2005. Viscoelastic properties of short calf muscle-tendon units of older women: effects of slow and fast passive dorsiflexion stretches in vivo. Eur J Appl Physiol. 95, 131-9.

Hufschmidt, A., Mauritz, K.H., 1985. Chronic transformation of muscle in spasticity: a peripheral contribution to increased tone. J Neurol Neurosurg Psychiatry. 48, 676-85.

Inman, V.T., 1976 The joints of the ankle. Williams \& Wilkins, Baltimore.

Lamontagne, A., Malouin, F., Richards, C.L., 2000. Contribution of passive stiffness to ankle plantarflexor moment during gait after stroke. Arch. Phys. Med. Rehabil. 81, 351-8.

Lance, J.W., 1980. Symposium synopsis. In: Feldman, R.G., Young, R.R., Koella, W.P. (Eds), Spasticity: Disordered Motor Control, Year Book Medical Publishers, Chicago, pp. 485494.

McNair, P., Stanley, S., 1996. Effect of passive stretching and jogging on the series elastic muscle stiffness and range of motion of the ankle joint. Br. J. Sports Med. 30, 313-8.

McNair, P.J., Dombrowski, E.W., Hewson, D.J., Stanley, S.N., 2000. Stretching at the ankle joint: viscoelastic responses to holds and continuous passive motion. Med. Sci. Sports Ex. 33, 354-8. 
Narici, M.V., Maganaris, C.N., 2007. Plasticity of the muscle-tendon complex with disuse and aging. Ex. Sport Sci. Rev. 35, 126-34.

O'Driscoll, S.W., Giori, N.J., 2000. Continuous passive motion (CPM): theory and principles of clinical application. J. Rehabil. Res. Dev. 37, 179-188.

Rao, S.R., Saltzman, C.L., Wilken, J., Yak, H.J., 2006. Increased passive ankle stiffness and reduced dorsiflexion range of motion in individuals with diabetes mellitus. Foot Ankle Int. $27,617-22$.

Singer, B., Dunne, J., Allison, G., 2001. Clinical evaluation of hypertonia in the triceps surae muscles. Phys. Ther. Rev. 6, 71-80.

Singer, B.J., Dunne, J.W., Singer, K.P., Allison, G.T., 2003. Velocity dependent passive plantarflexor resistive torque in patients with acquired brain injury. Clin Biomech. 18, 15765.

Stein, T.P., Wade, C.E., 2005. Metabolic consequences of muscle disuse atrophy. J. Nutr. 135, 1824S-1828S.

Stolov W.C., The concept of normal muscle tone, hypotonia and hypertonia. Arch Phys Med Rehabil. 1966; 47(3):156-68.

Trevino, S.G., Buford, W.L. Jr, Nakamura, T., John Wright, A., Patterson, R.M., 2004. Use of a Torque-Range-of-Motion device for objective differentiation of diabetic from normal feet in adults. Foot Ankle Int. 25, 561-7.

Trudel, G., Uhthoff, H.K., 2000. Contractures secondary to immobility: Is the restriction articular or muscular? An experimental longitudinal study in the rat knee. Arch. Phys. Med. Rehab. 81, 6-13.

Yeh, C-Y., Chen, J-J., Tsai, K-H., 2007. Quantifying the effectiveness of the sustained muscle stretching treatments in stroke patients with ankle hypertonia. J. Electromyograp. Kinesiol. $17,453-61$

Williams, P.E., Goldspink, G., 1984. Connective tissue changes in immobilised muscle. J. Anat. 138, 343-50.

Zhang, L.Q., Chung, S.G., Bai, Z., Xu, D., van Rey, E.M., Rogers, M.W., Johnson, M.E., Roth, E.J., 2002 Intelligent stretching of ankle joints with contracture/spasticity. IEEE Trans Neural Syst Rehabil Eng. 10, 149-57. 